\title{
Therapeutic interventions efficacy on gross motor function in children with cerebral palsy
}

\author{
Eficácia de intervenções terapêuticas na função motora \\ grossa em crianças com paralisia cerebral
}

\section{Efectividad de las intervenciones terapéuticas sobre la función motora gruesa en niños con parálisis cerebral}

\author{
Ana Clara Santana de Souza ${ }^{1}$, Janaina Araujo Teixeira Santos ${ }^{1}$, \\ Alessandra Vidal Prieto ${ }^{1}$, Pollyana Helena Vieira Costa ${ }^{2}$, Rudney da \\ Silva ${ }^{3}$, Paulo José Barbosa Gutierres Filho ${ }^{1}$
}

1.Faculdade de Educação Física (FEF), Universidade de Brasília (UnB), Brasília-DF, Brasil.

2.Faculdade de Ciências Médicas de Minas Gerais (FCMMG), Belo Horizonte-MG, Brasil.

3.Centro de Ciências da Saúde do Esporte - Universidade do Estado de Santa Catarina, Florianópolis-SC, Brasil.

\section{Resumo}

Objetivo. Verificar a eficácia da intervenção terapêutica em crianças de 0 a 12 anos de idade, com paralisia cerebral, avaliada pela Medida da Função Motora Grossa. Método. PUBMed (MEDLINE), Cochrane, Web of Science, Scopus, Lilacs e PEDro. Seleção dos estudos foi realizada em duas fases. Fase 1: títulos e resumos de todas as referências identificadas foram exibidos independentemente para 2 investigadores. Fase 2: os mesmos pesquisadores aplicaram os critérios de inclusão para o texto completo dos artigos. Os principais dados de cada um dos estudos incluídos foram extraídos, como autor, ano de publicação, tamanho da amostra, características relevantes e conclusões sobre intervenções terapêuticas em crianças com paralisia cerebral. Resultado. 15 artigos foram combinados para análise qualitativa. A pontuação total da qualidade metodológica variou de 5 a 9 pontos. 14 estudos foram incluídos na metanálise. Os resultados demonstraram uma variação significante quando comparados à terapia convencional realizada através da fisioterapia no escore total e no subgrupo de treinamento, mas não na hipoterapia, tarefas de atividade e outras terapias. Conclusão. As terapias comumente aplicadas em crianças com PC, quando integradas a tarefas funcionais, parecem ter melhores resultados quando comparadas à fisioterapia convencional.

Unitermos. Paralisia cerebral; Saúde da criança; Terapêutica; Revisão sistemática; Metanálise

\footnotetext{
Abstract

Objective. Verify therapeutic intervention efficacy in children between the ages of 0 to 12 , with cerebral palsy, evaluated by Gross Motor Function Measure. Method. PUBMed (MEDLINE), Cochrane, Web of Science, Scopus, Lilacs and PEDro. During phase 1, titles and abstracts of all identified references were independently displayed to 2 investigators. In phase 2, the same researchers applied the inclusion criteria for complete articles text. Key data from each of the included studies were extracted, such as author, year of publication, sample size, relevant characteristics, and conclusions on therapeutic interventions in children with cerebral palsy. Results. 15 articles were combined for qualitative analysis. Total scores for methodological quality ranged from 5 to 9 points. Covering the included articles for qualitative analysis, 14 studies were included in meta-analysis. Results demonstrated a significant variation when
} 
compared to conventional therapy performed through physical therapy in total scores and subgroup training, but not in hippotherapy, activity tasks and other therapies. Conclusion. Commonly therapies applied to children with CP when integrated with functional tasks appear to have better results when only compared to conventional physiotherapy.

Keywords. Cerebral palsy; Child health; Therapeutics; Systematic Review; Meta-analysis

\begin{abstract}
Resumen
Objetivo. Verificar la efectividad de la intervención terapéutica en niños de 0 a 12 años con parálisis cerebral, evaluados mediante la Medida de Función Motora Gruesa. Método. PUBMed (MEDLINE), Cochrane, Web of Science, Scopus, Lilacs y PEDro. La selección de estudios se llevó a cabo en dos fases. Fase 1: los títulos y resúmenes de todas las referencias identificadas se mostraron de forma independiente para 2 investigadores. Fase 2: los mismos investigadores aplicaron los criterios de inclusión para el texto completo de los artículos. Se extrajeron los datos principales de cada uno de los estudios incluidos, como autor, año de publicación, tamaño de la muestra, características relevantes y conclusiones sobre intervenciones terapéuticas en niños con parálisis cerebral. Resultado. se combinaron 15 artículos para el análisis cualitativo. La puntuación total de la calidad metodológica osciló entre 5 y 9 puntos. Se incluyeron 14 estudios en el metanálisis. Los resultados mostraron una variación significativa en comparación con la terapia convencional realizada mediante fisioterapia en la puntuación total y en el subgrupo de entrenamiento, pero no en hipoterapia, tareas de actividad y otras terapias. Conclusión. Las terapias que se aplican comúnmente a los niños con parálisis cerebral, cuando se integran con tareas funcionales, parecen tener mejores resultados en comparación con la fisioterapia convencional.
\end{abstract}

Palabras clave. Parálisis cerebral; Salud de los niños; Terapia; Revisión sistemática; Metaanálisis

Research developed at Universidade de Brasília (UnB), Brasília-DF, Brasil.

Corresponding author: Ana Clara S. de Souza. R. Stela de Souza 281/301. Sagrada Família. Belo Horizonte-MG, Brasil. Telefone: (31) 98516-4232. E-mail: ac.reabilitacaoinfantil@gmail.com

\title{
INTRODUCTION
}

Cerebral palsy (CP) is a collection of clinical symptoms that affect a child's neurological and motor development throughout life ${ }^{1}$ and which can lead to a number of negative results $^{1,2}$. Individuals with $\mathrm{CP}$ present changes in tone, posture and movement, leading to daily living activity limitations, such as transportation, food and personal care. Cerebral palsy affects about 2 children per 1000 live births worldwide, becoming the most common cause of severe physical disability during childhood ${ }^{2}$. There is lack of studies in Brazil that prove specific investigations of prevalence and 
incidence of CP in the national scenario, however, based on data demonstration from other countries, there are predominance projections ${ }^{3}$ of CP in developing countries. In developed countries the prevalence found varies from 1.5 to $5.9 / 1000$ live births; it is estimated that the incidence of CP in developing countries is $7 / 1000$ live births ${ }^{4}$, and data shown estimates around 30,000 to 40,000 of new cases per year ${ }^{5}$.

However, despite being one of the most prevalent damaging conditions in most parts of the globe ${ }^{2}$ the efficacy of therapeutic treatment in children with cerebral palsy has shown complexity when trying to be proven scientifically in result of the low methodological quality of studies ${ }^{6}$. Studies on therapeutic interventions in children with cerebral palsy evaluated the efficacy of treatments that applied Neurodevelopment Therapy (NDT ${ }^{7}$ ), strength training ${ }^{8}$ upper limbs $^{9}$ interventions ${ }^{6}$, orthoses ${ }^{10}$ usage $^{11}$, and varied interventions ${ }^{12}$. Sequentially, interventions were studied, such as, induced restraint therapy ${ }^{13}$, efficacy of physical therapy in postural control $^{14}$, passive stretching ${ }^{15}$, hydrotherapy ${ }^{16}$, hippotherapy ${ }^{17}$, and orthoses ${ }^{18}$ besides, a variety of other interventions. Most recent revisions have studied therasuit ${ }^{19}$, therapeutic exercises ${ }^{20}$, surgical interventions ${ }^{21}$, hipotherapy ${ }^{22}$, a variety of therapies focusing on gait ${ }^{23}$.

In such context, one of the ways of evaluating the efficacy of various therapeutic treatments applied to children with cerebral palsy has been quantitative analysis of gross 
motor function. Among current existing scales for such condition $^{18}$, one of the most used is the GMFM ${ }^{24}$. The GMFM operates with an evaluation scale responsible for quantifying global motor function without considering the quality performance, constructed especially for children with PC 25 and sufficiently applied in scientific reviews for this population. Considered accurate interventions with positive results are the Treadmill training and Kinesiotherapy $6,8,12,14,20$; treatments that need more scientific proof, in result that can diverge, are hippotherapy and Virtual Training - also known as Game therapy; Neurodevelopment Therapy (NDT) in several studies did not obtain improvement in the gross motor function.

There is still a major number of interventions that appear in lesser quantity in scientific reviews, yet it is necessary to study to evaluate its efficiency, stating its reproducibility or not; a few are: Vestibular Rehabilitation, Orthoses usage and Group Training. The objective of this systematic review was to verify the efficacy of therapeutic interventions in children, aged 0 to 12 years, with cerebral palsy and evaluated through the GMFM 66 and/or 88.

\section{METHOD}

\section{Protocol Register}

The protocol systematic review was registered on the International Prospective Register of Systematic Reviews (PROSPERO) under the number CRD42016051560 and 
followed the recommendations proposed by the Preferred Reporting Items for Systematic Review and Meta-analyses: The PRISMA Statement ${ }^{26}$.

\section{Eligibility Criteria}

Inclusion Criteria

Randomized controlled trials were included and in which authors investigated the effects of therapeutic interventions in children with cerebral palsy among the ages of 0 to 12 years. Only articles that used quantitative evaluation of gross motor function as an instrument were used, Measure of Motor Gross ${ }^{24}$ Function (GMFM 66 and/or 88) in Pre and post intervention in control group. The option of selection of studies using GMFM 66 and/or 88 is justified because this observation instrument is standardized, developed, and validated to measure gross motor function changes that occurs over time in children with $\mathrm{PC}^{25}$ and is widely used in scientific reviews for this population. All language types were included with no restriction on the date of publication.

\section{Exclusion Criteria}

Studies with children with cerebral palsy were excluded and with previous accompanying diagnosis of muscular dystrophy or neurodegenerative diseases, studies involving individuals aged over 12 years of age, using electrical stimulation therapy, biofeedback, behavioral and/or educational therapy, with botulinum toxin, dorsal selective rhizotomy, osteotomies and/or other surgeries and 
orthopedic procedures, pharmaceutical intervention, dental and oral motor control studies, involving nutrition, acupuncture, psychology, applied physiology, in this specific case, utilizing hyperbaric oxygen.

\section{Information Source}

Electronic database selected were: PUBMed (MEDLINE), Cochrane, Web of Science, Scopus, Lilacs and PEDro. Search strategy included descriptors proposed in the Medical Subject Headings (MeSH) referring to cerebral palsy: "Cerebral palsy", to the child: "Child", "Children", therapeutic intervention: "Intervention", "Interv Sch Clin" Therapeutics "," Therapeutic "," Gross "," Motor "," Function "," Physiology "associated with a sensitive list of key terms for clinical trials in March 2018.

Combination of keywords:

1 "Cerebral palsy" AND "Child OR Children" AND "Intervention OR Interv Sch Clin OR Therapeutics OR Therapeutic"

2 "Cerebral palsy" AND "Child OR Children" AND "Intervention OR Interv Sch Clin OR Therapeutics OR Therapeutic" AND "Gross Motor Function"

3 "Cerebral palsy" AND "Child OR Children" AND "Intervention OR Interv Sch Clin OR Therapeutics OR Therapeutic" AND "Gross Motor Function" AND "Physiology" 


\section{Research}

EndNote software tool was used for archive management aiming on the identification and control of bibliographic references, mainly regarding the potential of duplicity of scientific articles existing in different databases.

\section{Study Selection}

Final articles were selected through a two-step process. In phase 1 , the titles and abstracts of all identified references were independently displayed for two researchers. During phase 1, they excluded articles that did not meet the inclusion criteria. In phase 2 , the same researchers applied the inclusion criteria for full text articles. In cases of discrepancy the third reviewer was consulted for a final decision.

\section{Data Extraction}

The first author performed the extraction of data from included articles, and a second author checked all the collected information. Once again, in case of discrepancy the third reviewer was consulted for final decision. The authors extracted from each of the included studies key-data: author, year of publication, sample size, relevant characteristics, and conclusions on therapeutic interventions in children with cerebral palsy. 


\section{Risk Bias and Methodological Quality Assessment}

Risk bias and methodological quality of included clinical trials were independently assessed by two reviewers using PEDro scale, which is based on the Delphi list developed by Verhagen et al. (1998) ${ }^{27}$. PEDro score ranges from 1 point (without quality) to 10 points (excellent quality). Disagreements were resolved by consensus or a third-party review.

\section{Measure Summary}

The only studies which were considered were studies in which results were measured by the Measure of Gross Motor Function (GMFM 66 and/or 88). Consequently, we analyzed the measures of gross motor function results of articles included for review, which should include the data of the five GMFM dimensions: "A: Lying and rolling". "B: Sit", "C: Crawl and Kneel", "D: Standing", "E: Walk, Run and Jump". The GMFM score used in the selected articles is standardized by means of a guide containing all guidelines of all tests involved and ranges from 0 to 3,0 being the progress which does no initiate and 3 which is totally complete. The higher the score, the better the child's performance.

\section{Statistical Analysis}

The included studies were considered to have utilized the same assessment measure, the mean difference (measures the absolute difference between the mean values 
in two groups in a clinical trial), and 95\% confidence intervals were considered in the meta-analysis procedure. The necessary data to calculate the mean difference (MD) for continuous results were: mean change (baseline for followup); Standard Deviation (SD) of the mean difference; Number in each comparison group ( $n$ ) at the time of intervention. To calculate the mean change in a variable from the baseline for follow-up it was used: mean difference = mean at follow-up less mean at baseline. The same process was used to calculate the mean difference in the experimental and control groups. The authors were contacted for lack of data. In case of no response, data were not included in the analysis. Due to the omission of statistical heterogeneity, we verified the results using the fixed effects mode. The heterogeneity of the studies was evaluated by the statistics of I2 and 95\% CI. Statistical analysis was performed using the Review Manager software version 5.3.

\section{RESULTS}

\section{Study Selection}

Out of 770 studies identified in the 6 electronic databases, 477 remained after removing the duplicate articles. During phase 1 (screening phase), a comprehensive evaluation of the abstracts was carried out, which excluded 432 articles, resulting in 45 studies. 24 additional articles were identified in the reference lists of the selected studies, but none met all the inclusion criteria. Subsequently, 30 
studies were excluded for several reasons (Figure 1). Lastly, only fifteen articles were included. Figure 1 shows the flowchart describing the process of identification, inclusion and exclusion of studies.

Figure 1. Flowchart.

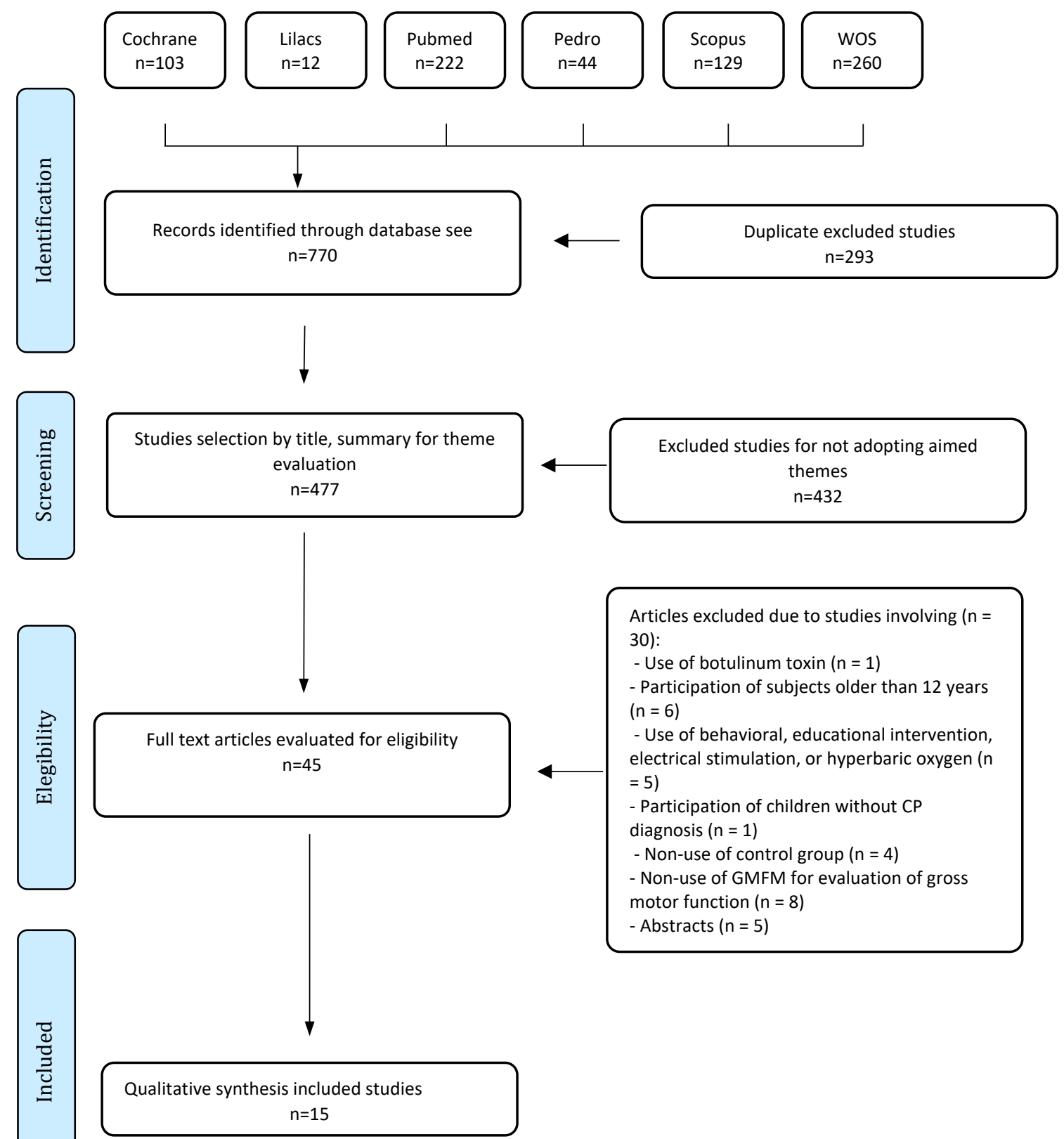




\section{Study Characteristics}

Of fifteen selected studies, three articles were from Taiwan 28-30, three were carried out in South Korea ${ }^{31-33}$, and another eight were conducted in several countries: one in Australia $^{34}$, one in Saudi Arabia ${ }^{35}$, one in Belgium ${ }^{36}$, one in Brazil $^{37}$, one in Canada ${ }^{38}$, one in Sweden ${ }^{39}$, one in China ${ }^{40}$, one in Israel ${ }^{41}$, and one in Italy ${ }^{42}$. The size of the sample varied from 549 to 7144 . Researchers from three studies evaluated "Activity-Focused Task" as an intervention 38,39,41, three other groups of researchers studied "hippotherapy" as an intervention $28,33,34$, two evaluated "Treadmill Training"35,37, a group studied "Game Therapy"28, one evaluated "Group Physical Therapy"36, one investigated "Hydrotherapy"29, one studied "Functional Training"32, one evaluated "Walking from Sitting to Standing"30, a group studied "Daytime Use of Orthoses" and a group studied "Vestibular Training". Table 1 summarizes the descriptive characteristics of the studies.

\section{Risk Bias Study}

The quality of included studies is summarized in Table 2. The total scores for methodological quality vary from 5 to 9 points, meaning that two scored 5 points 33,39 , two 6 points 28,32 , four 7 points $29,30,35,36$, six 8 points $34,37,38,40,41$, and one 9 points ${ }^{31}$. 
Table 1. Descriptive characteristics of included studies.

\begin{tabular}{|c|c|c|c|c|c|c|c|c|c|c|}
\hline Year & Author & Country & Age & $\begin{array}{c}\text { Intervention } \\
\text { (EG) }\end{array}$ & $\begin{array}{c}\text { Intervention } \\
\text { (CG) }\end{array}$ & $\begin{array}{c}\text { Sample } \\
\text { (EG) }\end{array}$ & $\begin{array}{c}\text { Sample } \\
\text { (CG) }\end{array}$ & Instruments & Frequency & Results \\
\hline 2010 & $\begin{array}{l}\text { Bar- } \\
\text { Haim S } \\
\text { et al. }{ }^{41}\end{array}$ & Israel & $\begin{array}{l}6-12 \\
\text { years }\end{array}$ & Motor training & $\begin{array}{l}\text { Neurodevelopmental } \\
\text { treatment (NDT) }\end{array}$ & 38 & 38 & GMFM 66 & $\begin{array}{l}\text { 1h/day; } 3 \\
\text { days/week; } \\
12 \text { weeks }\end{array}$ & $\begin{array}{l}\text { Gross Motor } \\
\text { Function } \\
\text { Improvement }\end{array}$ \\
\hline 2013 & $\begin{array}{l}\text { Chen } \\
\text { CL et } \\
\text { al. } .^{28}\end{array}$ & Taiwan & $\begin{array}{l}6-12 \\
\text { years }\end{array}$ & $\begin{array}{l}\text { Cycling Virtual } \\
\text { Training }\end{array}$ & Aerobics & 13 & 14 & GMFM 66 & $\begin{array}{l}40 \text { min/day; } \\
3 \text { day/week; } \\
12 \text { weeks }\end{array}$ & $\begin{array}{l}\text { No significant } \\
\text { difference }\end{array}$ \\
\hline 2009 & $\begin{array}{l}\text { Davis E } \\
\text { et al. }{ }^{34}\end{array}$ & Australia & $\begin{array}{l}4-12 \\
\text { year }\end{array}$ & $\begin{array}{l}\text { Equine } \\
\text { Therapy }\end{array}$ & $\begin{array}{l}\text { Conventional } \\
\text { Physiotherapy }\end{array}$ & 35 & 37 & GMFM 66 & $\begin{array}{l}30-40 \\
\text { min/day; } 1 \\
\text { day/week; } \\
10 \text { weeks }\end{array}$ & $\begin{array}{l}\text { No significant } \\
\text { difference }\end{array}$ \\
\hline 2016 & $\begin{array}{l}\text { Emara } \\
\text { HA et } \\
\text { al. }{ }^{35}\end{array}$ & $\begin{array}{l}\text { Saudia } \\
\text { Arabia }\end{array}$ & $\begin{array}{l}6-8 \\
\text { years }\end{array}$ & $\begin{array}{l}\text { Treadmill } \\
\text { Training + } \\
\text { Therapeutic } \\
\text { exercices }\end{array}$ & $\begin{array}{l}\text { Suspended exercices } \\
+ \text { Therapeutic } \\
\text { exercices }\end{array}$ & 10 & 10 & GMFM 88 & $\begin{array}{l}3 \\
\text { days/week; } \\
12 \text { weeks }\end{array}$ & $\begin{array}{l}\text { GMFM } \\
\text { dimensions D } \\
\text { and } \mathrm{E} \\
\text { improvement }\end{array}$ \\
\hline 2014 & $\begin{array}{l}\text { Franki I } \\
\text { et al. }{ }^{36}\end{array}$ & Belgium & $\begin{array}{l}4-9 \\
\text { years }\end{array}$ & $\begin{array}{l}\text { Group } \\
\text { Physiotherapy }\end{array}$ & $\begin{array}{l}\text { Individual } \\
\text { Physiotherapy }\end{array}$ & 5 & 5 & GMFM 88 & $\begin{array}{l}40 \text { min/day; } \\
3 \\
\text { days/week; } \\
10 \text { weeks }\end{array}$ & $\begin{array}{l}\text { No significant } \\
\text { difference }\end{array}$ \\
\hline 2013 & $\begin{array}{l}\text { Grecco } \\
\text { Let } \\
\text { al. }{ }^{37}\end{array}$ & Brazil & $\begin{array}{l}-12 \\
\text { years }\end{array}$ & $\begin{array}{l}\text { Treadmill } \\
\text { training }\end{array}$ & $\begin{array}{l}\text { Gait and ground } \\
\text { training }\end{array}$ & 16 & 17 & GMFM 88 & $\begin{array}{l}30 \text { min/day; } \\
2 \\
\text { days/week; } \\
7 \text { weeks }\end{array}$ & $\begin{array}{l}\text { GMFM } \\
\text { dimensions C, } \\
D \text { and } \mathrm{E} \\
\text { improvement }\end{array}$ \\
\hline 2015 & $\begin{array}{l}\text { Kwon J } \\
\text { et al. }{ }^{31}\end{array}$ & $\begin{array}{l}\text { South } \\
\text { Korea }\end{array}$ & $\begin{array}{l}4-10 \\
\text { years }\end{array}$ & $\begin{array}{l}\text { Equine } \\
\text { therapy }+ \\
\text { conventional } \\
\text { physiotherapy }\end{array}$ & $\begin{array}{l}\text { Aerobics }+ \\
\text { Conventional } \\
\text { Physiotherapy }\end{array}$ & 45 & 46 & GMFM 88 & $\begin{array}{l}30 \mathrm{~min} / \text { day; } \\
2 \\
\text { days/week; } \\
8 \text { weeks }\end{array}$ & $\begin{array}{l}\text { Gross Motor } \\
\text { Function } \\
\text { Improvement }\end{array}$ \\
\hline 2015 & $\begin{array}{l}\text { Lai C et } \\
\text { al. }{ }^{29}\end{array}$ & Taiwan & $\begin{array}{l}4-12 \\
\text { years }\end{array}$ & $\begin{array}{l}\text { Hydrotherapy } \\
+ \\
\text { Conventional } \\
\text { Physiotherapy }\end{array}$ & $\begin{array}{l}\text { Conventional } \\
\text { Physiotherapy }\end{array}$ & 11 & 13 & GMFM 66 & $\begin{array}{l}\text { 1h/day; } 1 \\
\text { day/week; } \\
12 \text { weeks }\end{array}$ & $\begin{array}{l}\text { Gross Motor } \\
\text { Function } \\
\text { Improvement }\end{array}$ \\
\hline 2011 & $\begin{array}{l}\text { Law C } \\
\text { et al. }{ }^{38}\end{array}$ & Canada & $\begin{array}{l}1-5 \\
\text { years }\end{array}$ & $\begin{array}{l}\text { Context- } \\
\text { focused } \\
\text { approach }\end{array}$ & $\begin{array}{l}\text { Child-centered } \\
\text { approach }\end{array}$ & 57 & 71 & GMFM 66 & 6 months & $\begin{array}{l}\text { No significant } \\
\text { difference }\end{array}$ \\
\hline 2015 & $\begin{array}{l}\text { Lee } \mathrm{M} \\
\text { et al. }{ }^{32}\end{array}$ & $\begin{array}{l}\text { South } \\
\text { Korea }\end{array}$ & $\begin{array}{l}5-10 \\
\text { years }\end{array}$ & $\begin{array}{l}\text { Progressive } \\
\text { functional } \\
\text { training }+ \\
\text { neurodevelop } \\
\text { mental } \\
\text { treatment } \\
\text { (NDT) }\end{array}$ & $\begin{array}{l}\text { Neuroevolutionary } \\
\text { Treatment (NDT) }\end{array}$ & 13 & 13 & GMFM 88 & $\begin{array}{l}\text { 30min/day; } \\
3 \\
\text { days/week; } \\
6 \text { weeks }\end{array}$ & $\begin{array}{l}\text { No significant } \\
\text { difference }\end{array}$ \\
\hline
\end{tabular}


Table 1 (cont.). Descriptive characteristics of included studies.

\begin{tabular}{|c|c|c|c|c|c|c|c|c|c|c|}
\hline Year & Author & Country & Age & $\begin{array}{l}\text { Intervention } \\
\text { (EG) }\end{array}$ & $\begin{array}{l}\text { Intervention } \\
\text { (CG) }\end{array}$ & $\begin{array}{l}\text { Sample } \\
\text { (EG) }\end{array}$ & $\begin{array}{l}\text { Sample } \\
\text { (CG) }\end{array}$ & Instruments & Frequency & Results \\
\hline 2007 & $\begin{array}{l}\text { Liao } \mathrm{HF} \\
\text { et al. }{ }^{30}\end{array}$ & Taiwan & $\begin{array}{l}5-12 \\
\text { years }\end{array}$ & $\begin{array}{l}\text { Sit and stand } \\
\text { training }+ \\
\text { conventional } \\
\text { physiotherapy }\end{array}$ & $\begin{array}{l}\text { Conventional } \\
\text { Physiotherapy }\end{array}$ & 10 & 10 & GMFM 88 & $\begin{array}{l}3 \times / \text { day; } 3 \\
\text { days/week; } \\
6 \text { weeks }\end{array}$ & $\begin{array}{l}\text { Gross Motor } \\
\text { Function } \\
\text { Improvement }\end{array}$ \\
\hline 2009 & $\begin{array}{l}\text { Lowing } \\
\text { K et } \\
\text { al. } .^{39}\end{array}$ & Sweden & $\begin{array}{l}1-6 \\
\text { years }\end{array}$ & $\begin{array}{l}\text { Objective- } \\
\text { oriented } \\
\text { funcional } \\
\text { therapy }\end{array}$ & $\begin{array}{l}\text { Task focused activity } \\
\text { training }\end{array}$ & 22 & 22 & GMFM 66 & $\begin{array}{l}1 \text { day/week; } \\
12 \text { weeks }\end{array}$ & $\begin{array}{l}\text { Gross Motor } \\
\text { Function } \\
\text { Improvement }\end{array}$ \\
\hline 2014 & $\begin{array}{l}\text { Park E } \\
\text { et al. } .^{33}\end{array}$ & $\begin{array}{l}\text { South } \\
\text { Korea }\end{array}$ & $\begin{array}{l}3-12 \\
\text { years }\end{array}$ & $\begin{array}{l}\text { Equine } \\
\text { Therapy }\end{array}$ & $\begin{array}{l}\text { Physiotherapy }+ \\
\text { Ocupational Therapy }\end{array}$ & 34 & 21 & $\begin{array}{l}\text { GMFM } 66+ \\
\text { GMFM } 88\end{array}$ & $\begin{array}{l}30 \text { min/day; } \\
1 \text { dia/week; } \\
8 \text { weeks }\end{array}$ & $\begin{array}{l}\text { GMFM } \\
\text { dimension E } \\
\text { improvement }\end{array}$ \\
\hline 2013 & $\begin{array}{l}\text { Zhao X } \\
\text { et al. }{ }^{40}\end{array}$ & China & $\begin{array}{l}1-4 \\
\text { years }\end{array}$ & $\begin{array}{l}\text { AFO dia (6-12 } \\
\text { hours) }+ \\
\text { conventional } \\
\text { physiotherapy }\end{array}$ & $\begin{array}{l}\text { AFO dia e noite (24 } \\
\text { hours) + } \\
\text { conventional } \\
\text { physiotherapy }\end{array}$ & 53 & 52 & GMFM 66 & 8 weeks & $\begin{array}{l}\text { Gross Motor } \\
\text { Function } \\
\text { Improvement }\end{array}$ \\
\hline 2017 & $\begin{array}{l}\text { Tramon } \\
\text { tano } \mathrm{M} \\
\text { et al. } 42\end{array}$ & Italy & $\begin{array}{l}3-11 \\
\text { years }\end{array}$ & $\begin{array}{l}\text { Vestibular } \\
\text { rehabilitation } \\
+ \\
\text { neurodevelop } \\
\text { mental } \\
\text { treatment } \\
\text { (NDT) }\end{array}$ & $\begin{array}{l}\text { Neuroevolutionary } \\
\text { treatment (NDT) }\end{array}$ & 7 & 7 & GMFM 88 & $\begin{array}{l}50 \text { min/day; } \\
2 x / \text { week; } \\
5 \text { weeks }\end{array}$ & $\begin{array}{l}\text { No significant } \\
\text { difference }\end{array}$ \\
\hline
\end{tabular}

Meta-Analysis Characteristics

Fourteen studies met the meta-analysis inclusion conditions and were tested by the fixed-effect model. The reported results of these studies were used to examine the effect of various therapies on gross motor function on individuals with cerebral palsy. The results confirmed a significant discrepancy when compared to conventional therapy in total scores and subgroup training, but not in 
hippotherapy, activity task and other therapies. In the present review, 4 forest plot (subgroups) were compiled which report I2 statistics (total [IC 95\%]) due to heterogeneity on continuous data (Figure 2).

Table 2. Methodological quality evaluation on studies included in the review analyzed through the PEDro scale.

\begin{tabular}{|c|c|c|c|c|c|c|c|c|c|c|c|c|}
\hline \multirow{2}{*}{ First Author, year } & \multicolumn{11}{|c|}{ Criteria } & \multirow{2}{*}{ Total } \\
\hline & $1 *$ & 2 & 3 & 4 & 5 & 6 & 7 & 8 & 9 & 10 & 11 & \\
\hline $\begin{array}{l}\text { Bar-Haim S et al., } \\
2010^{41}\end{array}$ & - & 1 & 1 & 1 & 1 & 0 & 0 & 1 & 1 & 1 & 1 & 8 \\
\hline Chen CL et al., $2013^{28}$ & - & 1 & 0 & 1 & 0 & 0 & 0 & 1 & 1 & 1 & 1 & 6 \\
\hline Davis E et al., $2009^{34}$ & - & 1 & 1 & 1 & 1 & 0 & 0 & 1 & 1 & 1 & 1 & 8 \\
\hline Emara HA et al., $2016^{35}$ & - & 1 & 0 & 1 & 0 & 0 & 1 & 1 & 1 & 1 & 1 & 7 \\
\hline Franki I et al., $2014^{36}$ & - & 0 & 1 & 1 & 0 & 0 & 1 & 1 & 1 & 1 & 1 & 7 \\
\hline Grecco L et al., $2013^{37}$ & - & 1 & 1 & 1 & 0 & 0 & 1 & 1 & 1 & 1 & 1 & 8 \\
\hline Kwon J et al', $2015^{31}$ & - & 1 & 1 & 1 & 1 & 0 & 1 & 1 & 1 & 1 & 1 & 9 \\
\hline Lai C et al., $2015^{29}$ & - & 1 & 0 & 1 & 0 & 0 & 1 & 1 & 1 & 1 & 1 & 7 \\
\hline Law C et al., $2011^{38}$ & - & 1 & 1 & 1 & 0 & 0 & 1 & 1 & 1 & 1 & 1 & 8 \\
\hline Lee $\mathrm{M}$ et al., $2005^{32}$ & - & 1 & 0 & 1 & 0 & 0 & 0 & 1 & 1 & 1 & 1 & 6 \\
\hline Liao HF et al., $2007^{30}$ & - & 1 & 1 & 1 & 0 & 0 & 1 & 0 & 1 & 1 & 1 & 7 \\
\hline Lowing $\mathrm{K}$ et al., $2009^{39}$ & - & 0 & 0 & 1 & 0 & 0 & 0 & 1 & 1 & 1 & 1 & 5 \\
\hline Park E et al., $2014^{33}$ & - & 0 & 0 & 1 & 0 & 0 & 0 & 1 & 1 & 1 & 1 & 5 \\
\hline Zhao X et al., $2013^{40}$ & - & 1 & 1 & 1 & 0 & 0 & 1 & 1 & 1 & 1 & 1 & 8 \\
\hline $\begin{array}{l}\text { Tramontano M et al., } \\
2017^{42}\end{array}$ & - & 1 & 1 & 1 & 0 & 0 & 1 & 1 & 1 & 1 & 1 & 8 \\
\hline
\end{tabular}

${ }^{*}$ Criteria not considered for final count because it is an item that evaluates the external validity ${ }^{26}$; Criteria 1, Eligible without punctuation; Criteria 2, Random allocation; Criteria 3, Hidden allocation; Criteria 4, Baseline measure; Criteria 5, Blind subjects; Criteria 6, Blind therapists; Criteria 7, Blind Evaluators; Criteria 8, <15\% withdrawal; Criteria 9, Treatment Intent; Criteria 10, Intergroup comparison; Criteria 11 , Precision and variability measure. 
Figure 2. Subgroups.

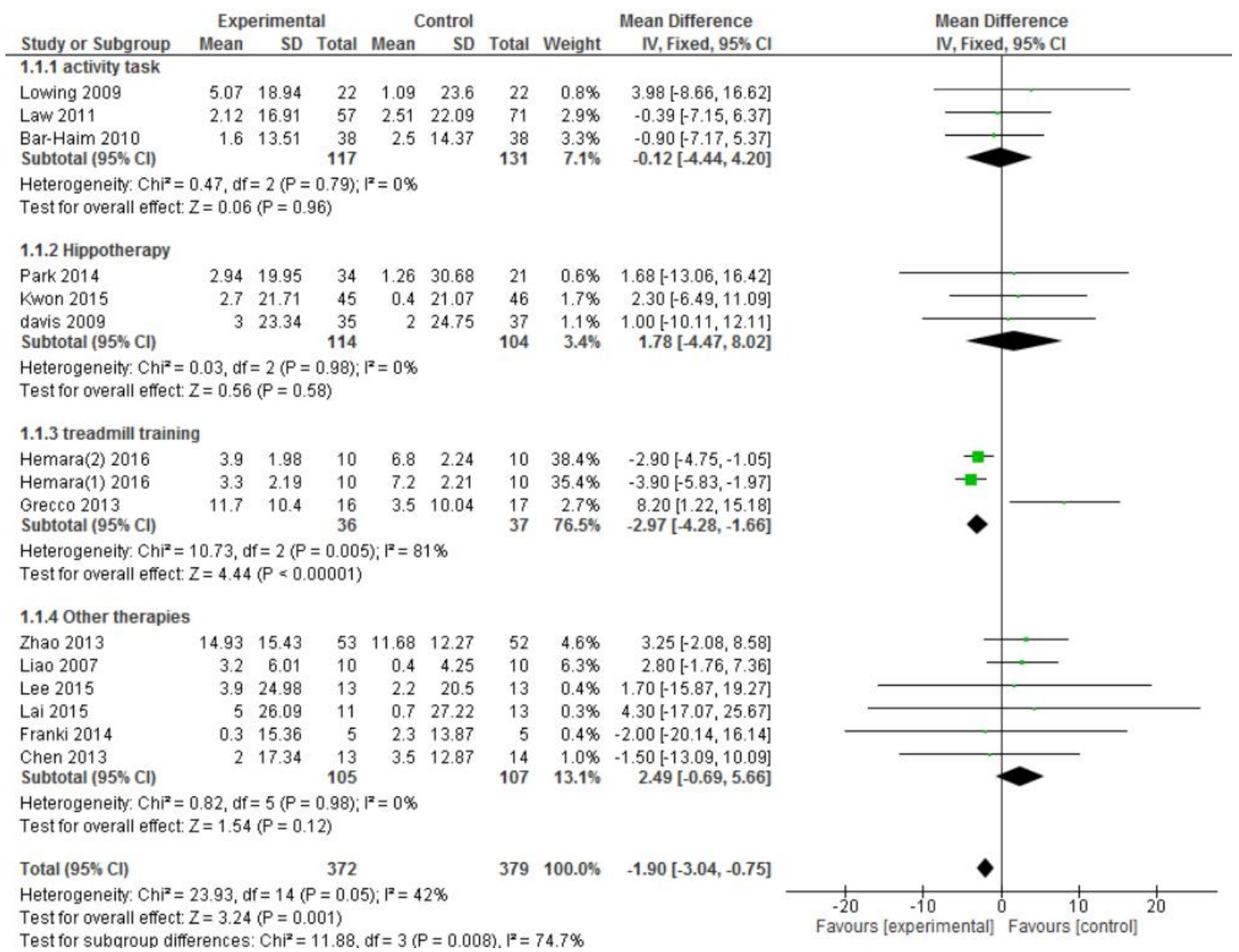

Three analysis did not present evidence on heterogeneity $(\mathrm{I} 2=0 \%)$ : activity tasks, hippotherapy and other therapies. One analysis presented high evidence of heterogeneity: treadmill training $(\mathrm{I} 2=81 \%)$. Heterogeneity I $2=42 \%$ (Figure 3 ).

\section{Activity Task}

Three studies measured the effects of task activity on gross motor function in individuals with cerebral palsy. 248 participants (117 experimental, 131 controls) were evaluated. The analysis did not show a significant 
improvement in the experimental group when compared to the control group ( $M D=-0.12$ (95\% CI -4.44 to 4.20), $\mathrm{p}=0.96 ; \mathrm{I} 2=0 \%$; Chi2 $\mathrm{p}=0.47)$.

Figure 3. Heterogeneity.

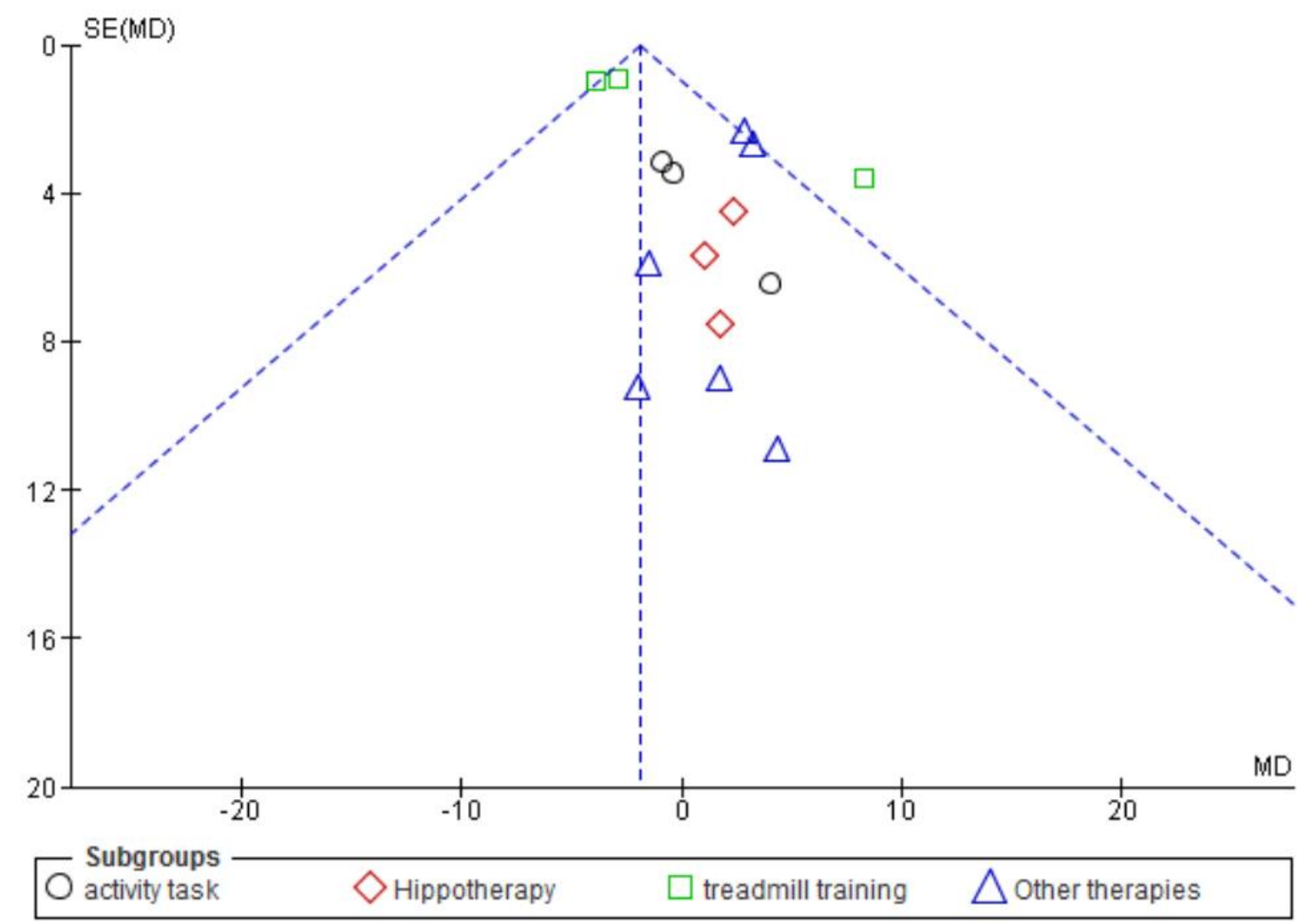

\section{Hippotherapy}

Three studies measured hippotherapy gross motor function effects in individuals with cerebral palsy. A total of 218 participants were evaluated (114 experimental; 104 control group). The analysis did not demonstrate improvement in the experimental group when compared to 
the control group ( $M D=1.78$ (IC $95 \%-4.47$ to 8.02 ); $\mathrm{p}=0.58 ; \mathrm{I} 2=0 \%$; Chi2 $\mathrm{p}=0.03)$.

\section{Treadmill Training}

Two studies (three variables) measured the treadmill training effects during gross motor function in individuals with cerebral palsy, 73 participants were evaluated (36 experimental; 37 control group). The analysis demonstrated significant improvement in the experimental group when compared to the control group (MD $=-2.97$ (IC 95\% -4.28 to $-1.66) ; p<0.00001 ; I 2=81 \%$; Chi2 $p=10.73)$.

\section{Other Therapies}

Six studies measured the effects of other therapies in individuals with cerebral palsy, which were: Virtual training (game therapy), Vestibular Rehabilitation, Orthoses usage, Functional Training, Hydrotherapy, Group Physiotherapy. A total of 212 participants were evaluated (105 experimental group; 107 control group). The analysis did not demonstrate significant improvement when compared to the control group $(M D=2.49$ (IC 95\% -0.69 to 5.66); $p=0.12 ; I 2=0 \%$; Chi2 $p=0.82$ ).

\section{Heterogeneity}

In the present review 4 portions of forest plots (subgroups) reported I2 statistics (total [IC 95\%]) due to the heterogeneity of the continuous data. Three analysis did not demonstrate indication of heterogeneity (I2=0\%): activity 
task, hippotherapy and other therapies. Treadmill training total $12=42 \%$ demonstrated high evidence of heterogeneity.

\section{DISCUSSION}

The present study focused on verifying, through gross motor function, the efficacy of current therapeutic treatments in specialized scientific reviews for the indicated population of children with cerebral palsy. The inclusion and exclusion criteria used allowed a broad search of relevant studies within the theme of the review. This review examined fifteen studies on therapeutic interventions in cerebral palsy. All articles included in this review were published as of 2007.

The quality of the studies, according to the Pedro Scale ranged from 5 to 9 points (only one appearing to have scored 9) indicating evidence of moderate to high for the effectiveness of interventions which achieved significant results. Nine different interventions were distinguished (equine therapy, hydrotherapy, treadmill training, functional training, orthotics, group physical therapy, virtual reality training, motor training, vestibular training) among the experimental groups. The outcome of the studies differed, yet it was possible to perform a meta-analysis. Most of the selected studies follow a purpose yet not only focused on body structure and function, but rather functional tasks, following the International Classification of Functioning, Disability and Health model, which addresses individuals with $\mathrm{CP}$ as biopsychosocial individuals ${ }^{43}$. 
Three 8 scoring studies, which analyzed motor training, treadmill training and orthoses $37,40,41$ usage obtained significant gross motor function improvement. On the other hand, three studies, also of quality 8 , and that investigated equine therapy, functional training and vestibular training $34,38,42$ did not obtain contrasting results from the control group, demonstrating that the intervention was not superior to conventional physiotherapy, child-centered approach and physical neuroevolutionary therapy. However, Kwon et al. (2015) ${ }^{31}$ demonstrated that equine therapy associated with conventional physiotherapy is superior to aerobic training associated with conventional physiotherapy in their high-evidence study, with a score of 937.

Treadmill training studies were carried out, in 2018, on children with cerebral palsy classified by the (GMFCS) II and III in the following variables: gait speed, endurance and lower limb muscle strength; after 6 weeks of intervention, reevaluations were observed, which demonstrated significant improvement in all measured ${ }^{44}$ variables. One study demonstrated that positive modifications were found in infants who underwent treadmill training twice a week during 25-minute sessions for 6 weeks ${ }^{45}$. Although the changes demonstrated being positive, the change in GMFM scores were not significant, different from what was found in the present review; but the children, classified in GMFCS I and II, did not have severe limitations; the studies evaluated by this review worked with children classified in GMFCS III ${ }^{35}$ and children in GMFCS I, II and III ${ }^{37}$. 
In 2006 the use of orthoses was also evaluated based on the GMFM; 20 children diagnosed with CP demonstrated significantly higher means with the use of orthosis in gross motor function and gait, reinforcing their prescription ${ }^{46}$. In addition to preventing deformities, orthoses align biomechanically certain body joints, which potentiates limb functions, explaining the best functional performance tasks, such as gait.

In a systematic review on equine therapy, twenty-two selected studies confirmed positive effects (except four that obtained non-significant or inconclusive results) on postural control and balance technique applied to individuals with $\mathrm{CP}$; however, the quality assessed by PEdro scale was $10 w^{47}$. In the current review, two out of three articles improved gross motor function ${ }^{37,33}$, and the PEdro scale ranged from 5 to 9 a better score than the review stated ${ }^{47}$.

In two 6-scoring studies there was no difference between the groups of virtual stationary bicycle training in relation to aerobic exercises and progressive functional training associated to neuro-evolutionary physiotherapy in relation to only neuro-evolutionary physiotherapy ${ }^{28,32}$. The low score of the stated studies can interfere in the internal validity of such study, possibly explaining the non-difference between groups. Referring to aerobic training, such improvement may be related to cardiorespiratory conditioning, and not to gross motor function; the GMFM is not a sensitive test for this outcome. 
Progressive training allows the re-adaptation of levels of training as the individual demonstrates improvement, promoting challenge and consequently better gain in training tasks ${ }^{32}$. In the evaluated study made by this review, findings demonstrated that functional training increased the thickness of lower limb muscles in individuals with $\mathrm{CP}$; but when the evaluation of gross motor function (GMFM) was restarted the results were higher but not statistically significant ${ }^{32}$. Such occurrence can be explained by the therapeutic application used in control and experimental groups (combined to functional training), neurodevelopmental treatment (NDT). NDT also uses in its principles, progressive muscular strengthening along with muscular stretches; Therapy was beneficial to both groups this might explain the lack of statistical difference between the two groups. Efficacy of neurodevelopmental intervention (NDT) in gross motor function parameters of children with cerebral palsy is already known ${ }^{32}$.

With the progress of physiotherapeutic interventions focused on functionality, virtual reality emerged as a therapy, which allows involvement and the presence of individuals in a stimulated daily life environment and their usual activities, thus providing motor learning. The virtual training was cited by one of the articles included in the present review, but it did not demonstrate significant difference with the control group, considering that no demonstration of certain functional activities that could resemble daily life $^{28}$ performed activities. Virtual training 
furnishes high potential for balance and gait recovery in various dysfunctions, including $\mathrm{CP}^{48}$. Training motivation with virtual reality is an essential factor for motor learning and may be an alternative for children with $\mathrm{CP}^{49}$.

Three studies scoring 7 on the Pedro Scale demonstrated superiority of treadmill, hydrotherapy and sitting and standing training when compared to suspended training and conventional physiotherapy. Interventions that simulate day-to-day functional activities, such as treadmill and sitting and standing training 30,35 , present better results because training is task-specific and performed with repetition, resulting in motor learning through brain neuroplasticity process.

Another 7-quality study did not demonstrate significant difference in its results when compared group physical therapy to individual physiotherapy ${ }^{36}$. The great majority of children with brain injury have specific limitations, and it is difficult to conduct joint training, since $\mathrm{CP}$ is a heterogeneous pathology and everyone has specific necessities, which in most cases do not apply to other individuals ${ }^{1,3}$.

Hydrotherapy, besides physical exercise, has certain benefits, such as to water physical principles, hydrostatic pressure, relative density, and buoyancy 29 . All these properties allow individuals with $\mathrm{CP}$ to live different experiences from those felt in a conventional physiotherapy session, increasing the repertoire of necessary movements and sensations for functional growth ${ }^{17}$. 
Two studies demonstrated that treadmill walking training associated with body weight support, performed three to four times a week for 12 weeks, was also proven to be significantly to movement and functional mobility capacity of children and adolescents with CP 35,50.

Functional training, such as sit and stand up, daily life, among other trainings are considered effective on functional performance improvement in children with cerebral palsy ${ }^{51}$. This can be explained based on motor learning theory; when working on replicable day to day activities, it is possible to obtain learning retention and by practicing it improves task performance.

In two 5-scoring studies, it was possible to note the superiority of objective-oriented functional training on tasks focused activities and equine therapy on conventional physiotherapy ${ }^{33,39}$. Different environment training and playing activities which simulate patients' real tasks have greater gain and demonstrate better results when compared to training focused only on structure and function. Physical therapy should be based on the individual's activity and participation ${ }^{48}$.

As noted, hydrotherapy, virtual reality, equine therapy, functional training, and treadmill training interventions can be aggregated to conventional workouts. Trainings focused on real environment or daily life performed activities tasks have greater probabilities of transferring from therapeutic environment to daily life activities, that is, when applying 
daily training one can maintain improvement on each therapeutic session.

Motor training, virtual reality, equine therapy, treadmill training, group physiotherapy, hydrotherapy, functional training, and orthotics usage are a wide range of possibilities that will not necessarily work for all individuals. The responsibility relies on a professional when evaluating which variables might interfere when choosing interventions and in the result of trainings, such as family characteristics, age, level of comorbidity, preferences, plasticity, acquisition of motor tasks and community ${ }^{52}$ access. There are still few good quality studies interested in physiotherapeutic interventions for individuals with CP.

\section{CONCLUSION}

It can be concluded that therapies commonly used in children with $\mathrm{CP}$ when integrated with functional tasks achieve better results when compared to conventional physiotherapy. Also, techniques that lead to an intensive training can improve technique efficacy that do not obtain significant difference with conventional training, becoming a suggestion for future studies.

\section{REFERENCES}

1.Rosenbaum P, Paneth N, Leviton MD, Goldstein M. Definition and classification of cerebral palsy. Dev Med Child Neurol 2007; 49:1-44. https://doi.org/10.1111/j.1469-8749.2007.00001.x 
2.Korzeniewski SJ, Slaughter J, Lenski M, Haak P, Paneth N. The complex aetiology of cerebral palsy. Nat Rev Neurol 2018;14:528-43. https://doi.org/10.1038/s41582-018-0043-6

3.Leite JMRS, do Prado GF. Paralisia cerebral Aspectos Fisioterapêuticos e Clínicos. Rev Neuroc 2004;12:41-5.

https://doi.org/10.4181/RNC.2004.12.41

4.Zanni G, Cemin NF, Peralles SN. Paralisia Cerebral: causas e prevalências. Fisioter Mov 2009;22:375-81.

https://periodicos.pucpr.br/index.php/fisio/article/view/19461/18801

5.Mancini MC, Alves ACM, Schaper C, Figueiredo EM, Sampaio RF, Coelho ZAC, et al. Gravidade da paralisia cerebral e desempenho funcional. Rev Bras Fisioter 2004;8:253-60.

6. Anttila H, Autti-Rämö I, Suoranta J, Mäkelä M, Malmivaara A. Effectiveness of physical therapy interventions for children with cerebral palsy: a systematic review. BMC Pediat 2008;8:14. https://doi.org/10.1186/1471-2431-8-14

7.Jones RA, Riethmuller A, Hesketh K, Trezise J, Batterham M, Okely AD. Promoting fundamental movement skill development and physical activity in early childhood settings: a cluster randomized controlled trial. Pediatr Exerc Sci 2011;23:600-15.

https://doi.org/10.1123/pes.23.4.600

8. Dodd KJ, Taylor NF, Damiano DL. A systematic review of the effectiveness of strength-training programs for people with cerebral palsy. Arch Phys Med Rehabil 2002;83:1157-64.

https://doi.org/10.1053/apmr.2002.34286

9.Boyd RN, Hays RM. Current evidence for the use of botulinum toxin type $A$ in the management of children with cerebral palsy: a systematic review. Eur J Neurol 2001;8(Suppl 5):1-20.

https://doi.org/10.1046/j.1468-1331.2001.00034.x

10.Morris C. A review of the efficacy of lower-limb orthoses used for cerebral palsy. Dev Med Child Neurol 2002;44:205-11. https://doi.org/10.1017/s0012162201001943

11.Teplicky R, Law M, Russel D. The effectiveness of casts, orthoses, and splints for children with neurological disorders. Infant Young Child 2002;15:42-50. https://doi.org/10.1097/00001163-20020700000007

12. Hur JJ. Review of research on therapeutic interventions for children with cerebral palsy. Acta Neurol Scand 1995;91:423-32. https://doi.org/10.1111/j.1600-0404.1995.tb00441.x

13. Hoare BJ, Wasiak J, Imms C, Carey L. Constraint-induced movement therapy in the treatment of the upper limb in children with hemiplegic cerebral palsy: a Cochrane systematic review. Clin Rehabil 2007;21:CD004149. https://doi.org/10.1177/0269215507080783

14. Harris SR, Roxborough L. Efficacy and effectiveness of physical therapy in enhancing postural control in children with cerebral palsy. Neural Plast 2005;12:229-43. https://doi.org/10.1155/NP.2005.229 15.Pin T, Dyke $P$, Chan M. The effectiveness of passive stretching in children with cerebral palsy. Dev Med Child Neurol 2006;48:855-62. https://doi.org/10.1017/S0012162206001836. 
16.Getz $M$, Hutzler $Y$, Vermeer A. Effects of aquatic interventions in children with neuromotor impairments: a systematic review of the literature. Clin Rehabil 2006;20:927-36.

\section{https://doi.org/10.1177/0269215506070693}

17.Sterba JA. Does horseback riding therapy or therapist-directed hippotherapy rehabilitate children with cerebral palsy? Dev Med Child Neurol 2007;49:68-73.

https://doi.org/10.1017/s0012162207000175.x

18. Autti-Rämö I, Suoranta J, Anttila H, Malmivaara A, Mäkelä M. Effectiveness of upper and lower limb casting and orthoses in children with cerebral palsy: an overview of review articles. Am J Phys Med Rehabil 2006;85:89-103.

https://doi.org/10.1097/01.phm.0000179442.59847.27

19. Almeida KM, Fonseca ST, Figueiredo PRP, Aquino AA, Mancini MC. Effects of interventions with therapeutic suits (clothing) on impairments and functional limitations of children with cerebral palsy: a systematic review. Braz J Phys Ther 2017;21:307-20. https://doi.org/10.1016/j.bjpt.2017.06.009

20.Ryan JM, Cassidy EE, Noorduyn SG, O'Connell NE. Exercise interventions for cerebral palsy. Cochrane Database Syst Rev 2017;6:CD011660.

https://doi.org/10.1002/14651858.CD011660.pub2

21.Galey SA, Lerner ZF, Bulea TC, Zimbler S, Damiano DL. Effectiveness of surgical and non-surgical management of crouch gait in cerebral palsy: a systematic review. Gait Posture 2017;54:93-105. 22.Rigby BR, Grandjean PW. The efficacy of equine-assisted activities and therapies on improving physical function. J Altern Complement Med 2016;22:9-24. https://doi.org/10.1016/j.gaitpost.2017.02.024 23. Moreau NG, Bodkin AW, Bjornson K, Hobbs A, Soileau M, Lahasky $\mathrm{K}$. Effectiveness of rehabilitation interventions to improve gait speed in children with cerebral palsy: systematic review and meta-analysis. Phys Ther 2016;96:1938-54. https://doi.org/10.2522/ptj.20150401 24. Lane M. Apostila do curso de Medição da Função Motora Grossa GMFM. Reabilitação Especializada São Paulo: GMFM; 2000.

25. Harries N, Kassirer M, Amichai T, Lahat E. Changes over years in gross motor function of 3-8 year old children with cerebral palsy: using the Gross Motor Function Measure (GMFM-88). Isr Med Assoc J 2004;6:408-11.

https://www.ima.org.il/FilesUploadPublic/IMAJ/0/52/26150.pdf

26. Moher D, Liberati A, Tetzlaff J, Altman DG; PRISMA Group. Preferred Reporting Items for Systematic Reviews and Meta-Analyses: The PRISMA Statement. Ann Intern Med 2009;151:264-9. https://doi.org/10.1371/journal.pmed.1000097

27.Verhagen AP, de Vet HC, de Bie RA, Kessels AG, Boers M, Bouter LM, et al. The Delphi list: a criteria list for quality assessment of randomized clinical trials for conducting systematic reviews developed by Delphi consensus. J Clin Epidemiol 1998;51:1235-41. https://doi.org/10.1016/s0895-4356(98)00131-0 
28.Chen CL, Chen CY, Liaw MY, Chung CY, Wang CJ, Hong WH. Efficacy of home-based virtual cycling training on bone mineral density in ambulatory children with cerebral palsy. Osteoporos Int 2013;24:1399-406. https://doi.org/10.1007/s00198-012-2137-0 29.Lai CJ, Liu WY, Yang TF, Chen CL, Wu CY, Chan RC. Pediatric aquatic therapy on motor function and enjoyment in children diagnosed with cerebral palsy of various motor severities. J Child Neurol 2015;30:200-8. https://doi.org/10.1177/0883073814535491 30.Liao HF, Liu YC, Liu WY, Lin YT. Effectiveness of loaded sit-to-stand resistance exercise for children with mild spastic diplegia: a randomized clinical trial. Arch Phys Med Rehabil 2007;88:25-31. https://doi.org/10.1016/j.apmr.2006.10.006

31.Kwon JY, Chang HJ, Yi SH, Lee JY, Shin HY, Kim YH. Effect of hippotherapy on gross motor function in children with cerebral palsy: a randomized controlled trial. J Altern Complement Med 2015;21:1521. https://doi.org/10.1089/acm.2014.0021

32. Lee $M$, Ko Y, Shin MMS, Lee $W$. The effects of progressive functional training on lower limb muscle architecture and motor function in children with spastic cerebral palsy. J Phys Ther Sci 2015;27:1581-4. https://doi.org/10.1589/jpts.27.1581

33.Park ES, Rha DW, Shin JS, Kim S, Jung S. Effects of hippotherapy on gross motor function and functional performance of children with cerebral palsy. Yonsei Med J 2014;55:1736-42. https://doi.org/10.3349/ymj.2014.55.6.1736

34.Davis E, Davies B, Wolfe R, Raadsveld R, Heine B, Thomason P, et al. A randomized controlled trial of the impact of therapeutic horse riding on the quality of life, health, and function of children with cerebral palsy. Dev Med Child Neurol 2009;51:111-9. https://doi.org/10.1111/j.1469-8749.2008.03245.x

35.Emara HA, El-Gohary TM, Al-Johany AA. Effect of body-weight suspension training versus treadmill training on gross motor abilities of children with spastic diplegic cerebral palsy. Eur J Phys Rehabil Med 2016;52:356-63.

https://www.minervamedica.it/en/getfreepdf/QnFkMEpQdXIOOTcvZIV WNDN6dndibIFTejFGNHZNOWk3RzV3REdMMHRVTVJjWXcreFJHbIpDU UtqSEU2dkxWOA\%253D\%253D/R33Y2016N03A0356.pdf

36. Franki I, Van den Broeck C, De Cat J, Tijhuis W, Molenaers $\mathrm{G}$, Vanderstraeten $\mathrm{G}$, et al. A randomized, single-blind cross-over design evaluating the effectiveness of an individually defined, targeted physical therapy approach in treatment of children with cerebral palsy. Clin Rehabil 2014;28:1039-52.

https://doi.org/10.1177/0269215514544984

37.Grecco LA, Zanon N, Sampaio LM, Oliveira CS. A comparison of treadmill training and overground walking in ambulant children with cerebral palsy: randomized controlled clinical trial. Clin Rehabil 2013;27:686-96.

https://doi.org/10.1177/0269215513476721

38. Law MC, Darrah J, Pollock N, Wilson B, Russell DJ, Walter SD, et al. Focus on function: a cluster, randomized controlled trial comparing 
child- versus context-focused intervention for young children with cerebral palsy. Dev Med Child Neurol 2011;53:621-9. https://doi.org/10.1111/j.1469-8749.2011.03962.x.

39.Löwing K, Bexelius A, Brogren Carlberg E. Activity focused and goal directed therapy for children with cerebral palsy - do goals make a difference? Disabil Rehabil 2009;31:1808-16.

https://doi.org/10.1080/09638280902822278

40.Zhao X, Xiao N, Li H, Du S. Day vs. day-night use of ankle-foot orthoses in young children with spastic diplegia: a randomized controlled study. Am J Phys Med Rehabil 2013;92:905-11. https://doi.org/10.1097/PHM.0b013e318296e3e8

41.Bar-Haim S, Harries N, Nammourah I, Oraibi S, Malhees W, Loeppky J, et al. Effectiveness of motor learning coaching in children with cerebral palsy: a randomized controlled trial. Clin Rehabil 2010;24:1009-20.

https://doi.org/10.1177/0269215510371428

42. Tramontano M, Medici A, Iosa M, Chiariotti A, Fusillo G, Manzari $L$, et al. The Effect of Vestibular Stimulation on Motor Functions of Children With Cerebral Palsy. Motor Control 2017;21:299-311. https://doi.org/10.1123/mc.2015-0089

43.Organização Mundial de Saúde (OMS)/Organização Panamericana de Saúde (OPAS). CIF classificação internacional de funcionalidade, incapacidade e saúde. Universidade de São Paulo; 2003. http://www.periciamedicadf.com.br/cif2/cif portugues.pdf

44. Hoffman RM, Corr BB, Stuberg WA, Arpin DJ, Kurz MJ. Changes in lower extremity strength may be related to the walking speed improvements in children with cerebral palsy after gait training. Res Dev Disabil 2018;73:14-20.

https://doi.org/10.1016/j.ridd.2017.12.005

45. De Torre CRMA. Efeitos do treino de marcha em esteira em crianças com paralisia cerebral (dissertação). São Carlos: Universidade Federal de São Carlos; 2012.

https://repositorio.ufscar.br/bitstream/handle/ufscar/5293/4361.pdf? sequence $=1$ \&isAllowed $=y \#: ~:$ : text=CONCLUS $\%$ C3\% $830 \% 3$ A $\% 200 \%$ 20treino\%20de\%20marcha, \%2C\%20II\%2C\%20III\%20e\%20IV.

46.Cury VCR, Mancini MC, Melo AP, Fonseca ST, Sampaio RF, Tirado MGA. The effects of the use of orthoses on the functional mobility of children with cerebral palsy. Rev Bras Fisioter 2006;10:65-74. https://doi.org/10.1590/S1413-35552006000100009

47. Moraes AG, Silva M, Copetti F, Abreu AC, de David AC. Hippotherapy in the postural control and balance in individuals with cerebral palsy: systematic review. Rev Neurocienc 2015;23:546-54. https://doi.org/10.4181/RNC.2015.23.04.1062.09p

48.Porras DC, Siemonsma P, Inzelberg $R$, Zeilig G, Plotnik $M$. Advantages of virtual reality in the rehabilitation of balance and gait: Systematic review. Neurology 2018;90:1017-25.

https://doi.org/10.1212/WNL.0000000000005603 
49.Kassee C, Caça C, Holmes MWR, Lloyd M. Home-based Nintendo Wii training to improve upper-limb function in children ages 7 to 12 with spastic hemiplegic cerebral palsy. J Pediatr Rehabil Med 2017;10:145-54. https://doi.org/10.3233/PRM-170439

50.Visser A, Westman M, Otieno S, Kenyon L. A Home-Based Body Weight-Supported Treadmill Program for Children With Cerebral Palsy: A Pilot Study. Pediatr Phys Ther 2017;29:223-9.

https://doi.org/10.1097/PEP.0000000000000406

51. Ketelaar M, Vermeer A, Hart H, Beek EP, Helders PJM. Effects of a functional therapy program on motor abilities of children with cerebral palsy. Phys Ther 2001;81:1534-44.

https://doi.org/10.1093/ptj/81.9.1534

52. Gannotti ME, Christy JB, Heathcock JC, Kolobe THA. A path model for evaluating dosing parameters for children with cerebral palsy. Phys Ther 2014;94:411-2. https://doi.org/10.2522/ptj.20130022 